\title{
A Spectral Gradient Projection Method for Sparse Signal Reconstruction in Compressive Sensing
}

\author{
Auwal Bala Abubakar ${ }^{1}$, Kanikar Muangchoo ${ }^{2}$, Auwal Muhammad ${ }^{3}$ \& Abdulkarim Hassan Ibrahim ${ }^{4}$ \\ ${ }^{1}$ Department of Mathematical Sciences, Faculty of Physical Sciences, Bayero University Kano, Kano, Nigeria \\ ${ }^{2}$ Faculty of Science and Technology, Rajamangala University of Technology Phra Nakhon, 1381, Pracharat 1 \\ Road, Wongsawang, Bang Sue, Bangkok 10800, Thailand \\ ${ }^{3}$ Department of Physics, Kano University of Science and Technology, Wudil, Kano, Nigeria \\ ${ }^{4}$ KMUTTFixed Point Research Laboratory, Room SCL 802 Fixed Point Laboratory, Science Laboratory \\ Building, Department of Mathematics, Faculty of Science, King Mongkut's University of Technology Thonburi, \\ Thrung Khru, Bangkok 10140, Thailand \\ Correspondence: Kanikar Muangchoo, Faculty of Science and Technology, Rajamangala University of \\ Technology Phra Nakhon (RMUTP), 1381, Pracharat 1 Road, Wongsawang, Bang Sue, Bangkok 10800, \\ Thailand.
}

Received: March 20, 2020

Accepted: April 27, 2020

Online Published: April 30, 2020

doi:10.5539/mas.v14n5p86

URL: https://doi.org/10.5539/mas.v14n5p86

\begin{abstract}
In this paper, a new spectral gradient direction is proposed to solve the $\ell_{1}$-regularized convex minimization problem. The spectral parameter of the proposed method is computed as a convex combination of two existing spectral parameters of some conjugate gradient method. Moreover, the spectral gradient method is applied to the resulting problem at each iteration without requiring the Jacobian matrix. Furthermore, the proposed method is shown to have converge globally under some assumptions. Numerically, the proposed method is efficient and robust in terms of its quality in reconstructing sparse signal and low computational cost compared to the existing methods.
\end{abstract}

Keywords: $\ell_{1}$-norm minimization, compressive sensing, signal reconstruction, spectral gradient method

\section{Introduction}

Consider the following unconstrained minimization problem for sparse signal recovery

$$
\min _{x}\left\{\frac{1}{2}\|A u-b\|_{2}^{2}+\mu\|u\|_{1}\right\}
$$

where $A \in \mathbb{R}^{m \times n}(m<<n), b \in \mathbb{R}^{m},\|\cdot\|_{1}$ is the $\ell_{1}$-norm of a vector $u \in \mathbb{R}^{n}$ usually called regularizer and $\mu \in \mathbb{R}^{+}$is a regularization parameter that can be interpreted as a trade-off between sparsity and residual error.

Several solvers have been proposed to solve the model (1). Due to simplicity and efficiency, iterative shrinkage thresholding (IST) and the quick iterative shrinkage thresholding algorithm (FISTA) are among methods used (Beck \& Teboulle, 2009; Khoramian, 2012). Additionally, in (Hale et al., 2007), a continuous search fixed point based method was introduced, and the Barzilai-Borwein stepsize (Huang \& Wan, 2017) implemented a nonmonotone line search acceleration technique. Gradient descent based methods are alternative methods used to solve model (1). Figueiredo (Figueiredo et al., 2007) initiate a gradient based method combined with projection to solve (1). Motivated by the Figueiredo method, Xiao and Zhu then suggested alternative method of solving the model (1) using the spectral gradient and the method of projection of the conjugate gradient (Xiao et al., 2011; Xiao \& Zhu, 2013). Unlike IST and FISTA, the model (1) was first reformulated to become a monotone system of equations. This reformulation procedure can be found in many literature (Ibrahim, Kumam, Abubakar, Abubakar, et al., 2020; Ibrahim, Kumam, Abubakar, Jirakitpuwapat, et al., 2020). Afterwards, an algorithm to solve the problem is then constructed (Abubakar, Rilwan, et al., 2020; Abubakar \& Kumam, 2018, 2019; Ibrahim et al., 2019). It should be noted that with the reformulation of the model (1) into a monotone equation system, the model (1) is now equivalent to the following nonlinear convex constrained equation,

$$
G(u)=0, u \in S,
$$


where $F: S \rightarrow \mathbb{R}^{n}$ is a continuous mapping and $S \subseteq \mathbb{R}^{n}$ is a convex set. Thus, solving (2) is equivalent to solving (1).

In this paper, we aim to present an innovative iterative approach for solving the compressive sensing problem of $\ell 1$-norm regularization. Motivated by the spectral parameters in the work of (Yuan et al., 2020) and (Amini et al., 2019), we propose a spectral gradient approach for solving the $\ell 1$ - regularized convex minimization problem (1) using the hyperplane projection technique. The spectral parameter is determined as a convex combination of the spectral parameters proposed in (Yuan et al., 2020) and (Amini et al., 2019) respectively. Under suitable conditions, the proposed method globally converges. Numerical results show that the proposed approach is effective and reliable compared to existing methods in terms of its efficiency in reconstructing sparse signal with low computational costs.

This paper is structured as follows: Section 1 introduce an algorithm to solve model (2) which is equivalent to solving (1). In Section 2, we establish the global convergence of the proposed algorithm. In Section 3, we illustrate the good practical behaviour our algorithm in reconstructing sparse signal. Finally, the last Section gives the conclusion.

\section{Algorithm}

This section begin by defining the projection map together with some appropriate assumptions. Finally a description of the proposed algorithm is given with some remarks.

Suppose $S$ is a nonempty, closed and convex subset of $\mathbb{R}^{n}$. Then for any $u \in \mathbb{R}^{n}$, its projection onto $S$, denoted by $P_{S}(u)$ is

The projection map is nonexpansive, that is

$$
P_{S}(u)=\operatorname{argmin}\{\|u-y\|: y \in S\} .
$$

$$
\left\|P_{S}(u)-P_{S}(y)\right\| \leq\|u-y\|, \forall u, y \in \mathbb{R}^{n} .
$$

Throughout we make the following assumptions

$\left(\mathrm{H}_{1}\right)$ The set of solution to (2), denoted by $\operatorname{Sol}_{G, S}$, is nonempty.

$\left(\mathrm{H}_{2}\right) F$ is monotone, that is,

$$
\langle G(u)-G(y),(u-y)\rangle \geq 0, \quad \forall u, y \in \mathbb{R}^{n} .
$$

$\left(\mathrm{H}_{3}\right) F$ is Lipschitz continuous, that is there exists $L>0$ such that

$$
\|G(u)-G(y)\| \leq L\|u-y\|, \forall u, y \in \mathbb{R}^{n} .
$$

Motivated by the work of (Abubakar, Kumam, et al., 2020) We propose the following search direction

$$
d^{k}:=\left(\begin{array}{ll}
-G^{k}, & k=0, \\
-\theta^{k} G^{k}, & k>0,
\end{array}\right.
$$

where $G^{k}=G\left(x^{k}\right)$ and $\theta^{k}$ is a convex combination of $\lambda_{*}^{k}$ and $\lambda_{* *}^{k}$ given respectively by

$$
\begin{gathered}
\theta^{k}=\tau^{k} \lambda_{*}^{k}+\left(1-\tau^{k}\right) \lambda_{* * \prime}^{k}, \\
\lambda_{*}^{k}=1-\frac{\left\langle G^{k}, d^{k-1}\right\rangle^{2}}{\left\|G^{2}\right\|^{2}\left\|d^{k-1}\right\|^{2}}, \lambda_{* *}^{k}=\frac{\left\|y^{k-1}\right\|^{2}}{\left\langle y^{k-1}, \tilde{s}^{k-1}\right\rangle}, \tau^{k} \in[0,1], \\
\tilde{s}^{k-1}=s^{k-1}+\left(\max \left(0,-\frac{\left\langle y^{k-1}, s^{k-1}\right\rangle}{\left\|y^{k-1}\right\|^{2}}\right)\right) y^{k-1}, s^{k-1}=u^{k}-u^{k-1}, y^{k-1}=G\left(u^{k}\right)-G\left(u^{k-1}\right) .
\end{gathered}
$$

The step by step implementation of our algorithm is illustrated below.

\subsection{Algorithm}

Step 0. Select $u^{0} \in S \subseteq \mathbb{R}^{n}, \epsilon_{0}, \sigma, \mu$, and $\tau^{k} \in(0,1)$. Set $k:=0$.

Step 1. Terminate whenever $\left\|G^{k}\right\| \leq \epsilon_{0}$. Else go to Step 2 .

Step 2. Find $d^{k}$ using (4)-(7).

Step 3. Find $\alpha^{k}=\mu^{i}$ where $i$ is the smallest non-negative integer such that

$$
-\left\langle G\left(u^{k}+\alpha^{k} d^{k}\right), d^{k}\right\rangle \geq \sigma \alpha^{k}\left\|d^{k}\right\|^{2} .
$$

Step 4. Find $z^{k}=u^{k}+\alpha^{k} d^{k}$. Terminate when $z^{k} \in S$ and $\left\|G\left(z^{k}\right)\right\| \leq \epsilon_{0}$. Else find

$$
u^{k+1}=P_{S}\left[u^{k}-\xi^{k} G\left(z^{k}\right)\right]
$$


where

$$
\xi^{k}=\frac{\left\langle\left(u^{k}-z^{k}\right), G\left(z^{k}\right)\right\rangle}{\left\|G\left(z^{k}\right)\right\|^{2}} .
$$

Step 5. Set $k=k+1$ and go to Step 1 .

2.2 Remark

By Cauchy-Schwartz inequality, we have

$$
0 \leq\left|\left\langle G^{k}, d^{k-1}\right\rangle\right| \leq\left\|G^{k}\right\|\left\|d^{k-1}\right\|,
$$

which implies

$$
0 \leq 1-\frac{\left\langle G^{k}, d^{k-1}\right\rangle^{2}}{\left\|G^{k}\right\|^{2}\left\|d^{k-1}\right\|^{2}} \leq 1
$$

Therefore, $\lambda_{*}^{k} \in(0,1)$.

\subsection{Remark}

Using definition of $\tilde{s}^{k-1}$ and $y^{k-1}$,

$$
\left\langle y^{k-1}, \tilde{s}^{k-1}\right\rangle \geq \max \left\{\left\langle y^{k-1}, s^{k-1}\right\rangle,\left\|y^{k-1}\right\|^{2}\right\} \geq\left\|y^{k-1}\right\|^{2}>0,
$$

which implies

$$
0 \leq \frac{\left\|y^{k-1}\right\|^{2}}{\left\langle y^{\left.k-1, \tilde{s}^{k-1}\right\rangle}\right.} \leq 1 .
$$

Therefore, $\lambda_{* *}^{k} \in(0,1)$.

\section{Global Convergence}

In this section, we use the following Lemmas to prove Theorem 3.5.

\subsection{Lemma}

Suppose that assumptions $\left(H_{1}\right)-\left(H_{3}\right)$ hold and the sequence $\left\{d^{k}\right\}$ is generated by Algorithm 2.1, then $\left\{d^{k}\right\}$ is sufficiently descent and bounded. That is

(i) $\left\langle G^{k}, d^{k}\right\rangle \leq-\left\|G^{k}\right\|^{2}$, and

(ii) $\left\|d^{k}\right\| \leq\left\|G^{k}\right\|, \forall k \geq 0$.

Proof. (i) For $k=0$,

$$
\left\langle G^{0}, d^{0}\right\rangle=-\left\|G^{0}\right\|^{2} .
$$

For $k \geq 1$, using the definition of $\theta^{k}$, Remark 2.2 and 2.3,

$$
\begin{aligned}
\theta^{k} & =\tau^{k}\left(1-\frac{\left\langle G^{k}, d^{k-1}\right\rangle^{2}}{\left\|G^{k}\right\|^{2}\left\|d^{k-1}\right\|^{2}}\right)+\left(1-\tau^{k}\right) \frac{\left\|y^{k-1}\right\|^{2}}{\left\langle y^{\left.k-1, \tilde{s}^{k-1}\right\rangle}\right.} \\
& \geq 0 .
\end{aligned}
$$

Therefore,

$$
\begin{aligned}
& \left\langle G^{k}, d^{k}\right\rangle=-\theta^{k}\left\|G\left(u^{k}\right)\right\|^{2} \\
& \leq 0,
\end{aligned}
$$

since $\theta^{k} \in(0,1)$. Hence, we have

$$
\left\langle G^{k}, d^{k}\right\rangle \leq-\left\|G^{k}\right\|^{2}, \forall k
$$

For the (ii) part, if $k=0$, then

$$
\left\|d^{0}\right\|=\left\|-G\left(u^{0}\right)\right\|=\left\|G^{0}\right\| .
$$

If $k \geq 1$, then using the definition of $d^{k}$, Remark 2.2 and Remark 2.3 we have,

$$
\begin{aligned}
& \left\|d^{k}\right\|=\left\|-\theta^{k} G^{k}\right\| \\
& =\left|\theta^{k}\right|\left\|G^{k}\right\| \\
& =\left(\tau^{k}\left(1-\frac{\left\langle G^{k}, d^{k-1}\right\rangle^{2}}{\left\|G^{k}\right\|^{2}\left\|d^{k-1}\right\|^{2}}\right)+\left(1-\tau^{k}\right) \frac{\left\|y^{k-1}\right\|^{2}}{\left\langle y^{k-1}, \tilde{s}^{k-1}\right\rangle}\right)\left\|G\left(u^{k}\right)\right\| \\
& \leq\left(\tau^{k}+\left(1-\tau^{k}\right)\right)\left\|G\left(u^{k}\right)\right\| \\
& =\left\|G^{k}\right\| .
\end{aligned}
$$




\subsection{Lemma}

Let assumptions $\left(H_{1}\right)-\left(H_{3}\right)$ be fulfilled, then there exists $\alpha^{k}=\mu^{i}$ satisfying (8) for some $i \in \mathbb{N} \cup\{0\}$ and $\forall k \geq 0$.

Proof. Let $k_{0} \geq 0$ such that (8) is not true for every non-negative integer $i$, that is,

$$
-\left\langle G\left(u^{k_{0}}+\mu^{i} d^{k_{0}}\right), d^{k_{0}}\right\rangle\left\langle\sigma \mu^{i}\left\|d^{k_{0}}\right\|^{2} .\right.
$$

Since $F$ is continuous, then allowing $i \rightarrow \infty$ in the above inequality,

$$
-\left\langle G\left(u^{k_{0}}\right), d^{k_{0}}\right\rangle \leq 0 .
$$

Likewise by (13), we have

$$
-\left\langle G^{k_{0}}, d^{k_{0}}\right\rangle>0 \text {. }
$$

This contradicts with (14).

\section{3 Lemma}

Suppose assumptions $\left(H_{1}\right)-\left(H_{3}\right)$ are fulfilled, $\left\{u^{k}\right\}$ and $\left\{z^{k}\right\}$ generated by Algorithm 2.1. Then

$$
\alpha^{k} \geq \max \left\{1, \frac{\mu\left\|G^{k}\right\|^{2}}{(L+\sigma)\left\|d^{k}\right\|^{2}}\right\} .
$$

Proof. Suppose $\alpha^{k} \neq 1$. Then $\alpha_{*}^{k}=\alpha^{k} \beta^{-1}$ does not satisfy (8), that is

$$
-\left\langle G\left(u^{k}+\alpha_{*}^{k} d^{k}\right), d^{k}\right\rangle<\sigma \alpha_{*}^{k}\left\|d^{k}\right\|^{2} .
$$

From Lemma 3.1(i) and assumption $\left(H_{3}\right)$,

$$
\begin{aligned}
\left\|G^{k}\right\|^{2} & \leq-\left\langle G^{k}, d^{k}\right\rangle \\
& \left.=\left\langle G\left(u^{k}+\alpha_{*}^{k} d^{k}\right)-F_{k}\right), d^{k}\right\rangle-\left\langle H\left(u^{k}+\alpha_{*}^{k} d^{k}\right), d^{k}\right\rangle \\
& \leq \alpha_{*}^{k}(L+\sigma)\left\|d^{k}\right\|^{2} .
\end{aligned}
$$

\section{Lemma 3.4}

Suppose that assumptions $\left(H_{1}\right)-\left(H_{3}\right)$ are fulfilled. If $\left\{u^{k}\right\}$ and $\left\{z^{k}\right\}$ are obtained from Algorithm 2.1, then $\left\{u^{k}\right\}$ and $\left\{z^{k}\right\}$ have bound. Moreover, $\lim _{k \rightarrow+\infty}\left\|u^{k}-z^{k}\right\|=0$.

Proof. Suppose that $\left\|G^{k}\right\| \neq 0$ and $\left\|G\left(z^{k}\right)\right\| \neq 0$ for all $k$. Then an infinite sequence is generated. From Step 3 of Algorithm 2.1

$$
\begin{aligned}
& \left\langle G\left(z^{k}\right),\left(u^{k}-z^{k}\right)\right\rangle=-\alpha^{k}\left\langle G\left(z^{k}\right), d^{k}\right\rangle \\
& \geq \sigma \alpha_{k}^{2}\left\|d^{k}\right\|^{2} \\
& =\sigma\left\|u^{k}-z^{k}\right\|^{2} \\
& >0 .
\end{aligned}
$$

If $\bar{u} \in \operatorname{Sol}_{G, S}$ such that $G(\bar{u})=0$, then by assumption $\left(H_{2}\right)$,

$$
\begin{aligned}
& \left\langle G\left(z^{k}\right),\left(u^{k}-\bar{u}\right)\right\rangle=\left\langle G\left(z^{k}\right),\left(u^{k}-z^{k}\right)\right\rangle+\left\langle G\left(z^{k}\right),\left(z^{k}-\bar{u}\right)\right\rangle \\
& \geq\left\langle G\left(z^{k}\right),\left(u^{k}-z^{k}\right)\right\rangle+\left\langle G(\bar{u}),\left(z^{k}-\bar{u}\right)\right\rangle \\
& =\left\langle G\left(z^{k}\right),\left(u^{k}-z^{k}\right)\right\rangle .
\end{aligned}
$$

From (3), (9), (16) and (17),

$$
\begin{aligned}
& \left\|u^{k+1}-\bar{u}\right\|^{2}=\left\|P_{X}\left[u^{k}-\xi^{k} G\left(z^{k}\right)\right]-P_{X}(\bar{u})\right\|^{2} \\
& \leq\left\|u^{k}-\xi^{k} G\left(z^{k}\right)-\bar{u}\right\|^{2} \\
& =\left\|u^{k}-\bar{u}\right\|^{2}-2 \xi^{k}\left\langle G\left(z^{k}\right),\left(u^{k}-\bar{u}\right)\right\rangle+\left\|\xi^{k} G\left(z^{k}\right)\right\|^{2} \\
& \leq\left\|u^{k}-\bar{u}\right\|^{2}-\frac{\left\langle G\left(z^{k}\right),\left(u^{k}-z^{k}\right)\right\rangle^{2}}{\left\|G\left(z^{k}\right)\right\|^{2}} \\
& \leq\left\|u^{k}-\bar{u}\right\|^{2}-\frac{\sigma^{2}\left\|G\left(z^{k}\right)\right\|^{2}\left\|u^{k}-z^{k}\right\|^{4}}{\left\|G\left(z^{k}\right)\right\|^{2}} \\
& =\left\|u^{k}-\bar{u}\right\|^{2}-\frac{\sigma^{2}\left\|u^{k}-z^{k}\right\|^{4}}{\left\|G\left(z^{k}\right)\right\|^{2}} .
\end{aligned}
$$

Thus, the sequence $\left\{\left\|u^{k}-\bar{u}\right\|\right\}$ is convergent since it is decreasing and bounded below, and therefore $\left\{u^{k}\right\}$ is also bounded. Also, by (18), 


$$
\left\|u^{k+1}-\bar{u}\right\|^{2} \leq\left\|u^{k}-\bar{u}\right\|^{2},
$$

which implies that $\forall k \geq 0$,

$$
\left\|u^{k}-\bar{u}\right\|^{2} \leq\left\|u^{0}-\bar{u}\right\|^{2} .
$$

Accordingly, by assumption $\left(H_{3}\right)$ and chosing $\gamma:=L\left\|u^{0}-\bar{u}\right\|$,

$$
\left\|G^{k}\right\|=\left\|G^{k}-G(\bar{u})\right\| \leq L\left\|u^{k}-\bar{u}\right\| \leq \gamma .
$$

By (16), assumption $\left(H_{2}\right)$ and Cauchy Schwartz inequality,

$$
\sigma\left\|u^{k}-z^{k}\right\| \leq \frac{\sigma\left\|\alpha^{k} d^{k}\right\|}{\left\|u^{k}-z^{k}\right\|} \leq \frac{\left\langle G\left(z^{k}\right),\left(u^{k}-z^{k}\right)\right\rangle}{\left\|u^{k}-z^{k}\right\|} \leq \frac{\left\langle G\left(u^{k}\right),\left(u^{k}-z^{k}\right)\right.}{\left\|u^{k}-z^{k}\right\|} \leq\left\|G\left(u^{k}\right)\right\|,
$$

which implies that

$$
\sigma\left\|u^{k}-z^{k}\right\| \leq\left\|G^{k}\right\| .
$$

Thus, from (19) we have that the sequence $\left\{z^{k}\right\}$ is bounded.

Now, by (18),

$$
\sigma^{2} \sum_{k=0}^{\infty}\left\|u^{k}-z^{k}\right\|^{4} \leq \sum_{k=0}^{\infty}\left(\left\|u^{k}-\bar{u}\right\|^{2}-\left\|u^{k+1}-\bar{u}\right\|^{2}\right)<\infty .
$$

which implies

$$
\lim _{k \rightarrow+\infty}\left\|u^{k}-z^{k}\right\|=0
$$

From (21), then

$$
\lim _{k \rightarrow+\infty} \alpha^{k}\left\|d^{k}\right\|=0
$$

Theorem 3.5

Suppose that assumptions $\left(H_{1}\right)-\left(H_{3}\right)$ are fulfilled. If the sequence $\left\{u^{k}\right\}$ is obtained from Algorithm 2.1, then

$$
\liminf _{k \rightarrow \infty}\left\|G^{k}\right\|=0 .
$$

Proof. Suppose to the contrary the relation (23) is untrue. Then there exist a positive constant $r_{2}$ such that $\forall k \geq 0$,

$$
\left\|G^{k}\right\| \geq r_{2}
$$

By relation (24) and Lemma 3.1 (i), we have that $\forall k \geq 0$,

$$
\left\|d^{k}\right\| \geq r_{2} \text {. }
$$

Multiplying both sides of (15) with $\left\|d^{k}\right\|$ together with (24) and Lemma 3.1 (ii), we get

$$
\begin{aligned}
\alpha^{k}\left\|d^{k}\right\| & \geq \max \left\{1, \frac{\left.\mu \| G^{k}\right) \|^{2}}{(L+\sigma)\left\|d^{k}\right\|^{2}}\right\}\left\|d^{k}\right\| \\
& \geq \max \left\{r_{2}, \frac{\mu r_{2}^{2}}{(L+\sigma) \gamma}\right\} .
\end{aligned}
$$

The above inequality contradicts with (22). Therefore (23) must hold.

\section{Numerical Experiment}

Reconstruction of sparse signal in compressive sensing is shown in this section to illustrate the performance of Algorithm 2.1. We compared Algorithm 2.1 to the following methods in the literature to demonstrate the efficiency of our proposed method in signal reconstruction: SGCS (Xiao et al., 2011), CGD (Xiao \& Zhu, 2013), and PSGM (Abubakar, Kumam, et al., 2020). In Matlab R2019b the four algorithms were programmed and run on a PC with a 2.40GHZ CPU processor and $8.00 \mathrm{~GB}$ RAM.

In this experiment, our aim is to recover a length $n$ sparse signal from a Gaussian noise sampling measurement of $m$, where the number of samples is normally smaller than the original signal. The efficiency of the restored signal is measured by squared error (MSE) mean:

$$
M S E=\frac{1}{n}\left\|\bar{u}-u^{*}\right\|^{2},
$$

where $\bar{u}$ is the original signal and $u^{*}$ is the signal restored. We set the signal size as $n=2^{12}, m=2^{10}$. The original signal $\bar{u}$ contains $2^{6}$ randomly nonzero elements. During the experiment, a random matrix $T$ is generated. This is done using the Matlab command rand(n,m). In addition, the observed date $e$ is computed by $e=T \bar{u}+j$, where $j$ is the Gaussian noise which we set as $N\left(0,10^{-4}\right)$. 
We set the parameters for Algorithm 2.1 as follows: $\rho=0.8, \sigma=10^{-4}, \tau^{k}=\frac{1}{\exp (k+1)^{k+1}}$. The parameters for the compared methods (SGCS, CGD, PSGM) are set as in their respective papers. In order to be fair in comparing the methods, we made use of the same initial point and terminate when $\frac{\left\|G^{k}-G^{k-1}\right\|}{\left\|G^{k-1}\right\|}<10^{-5}$, where $G^{k}=\frac{1}{2}\|e-T x\|_{2}^{2}+\eta\|u\|_{1}$ is the objective function. In what follows, we refer to Algorithm 2.1 as Algo.1.

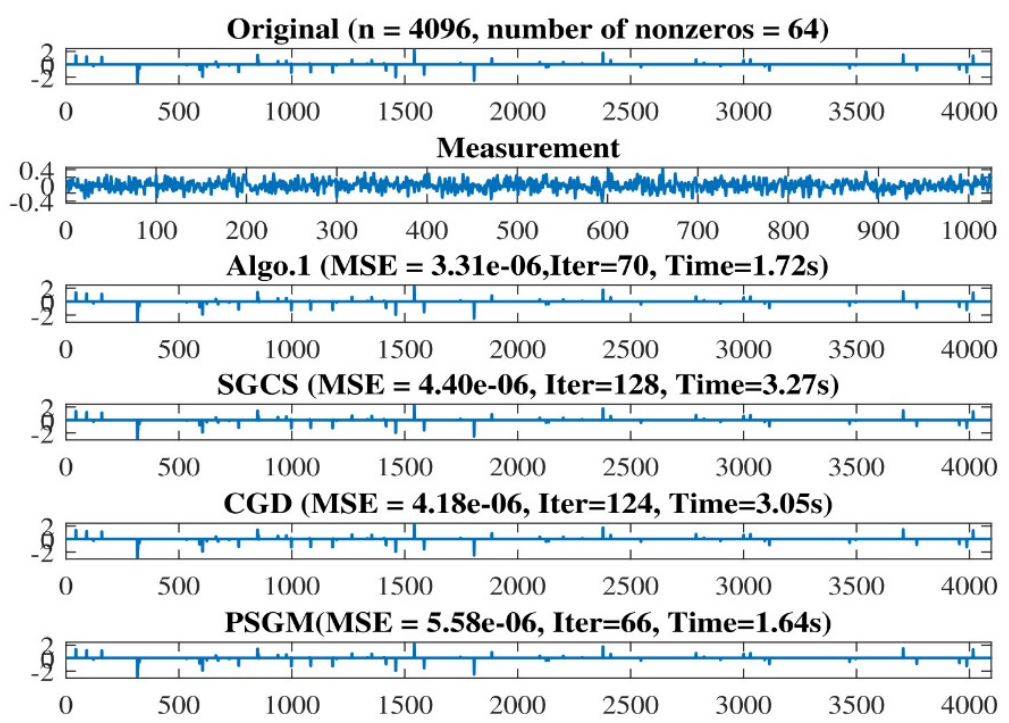

Figure 1. Sparse signal Reconstruction. From top to bottom: the original signal, the measurement and four algorithms of the reconstructed signals
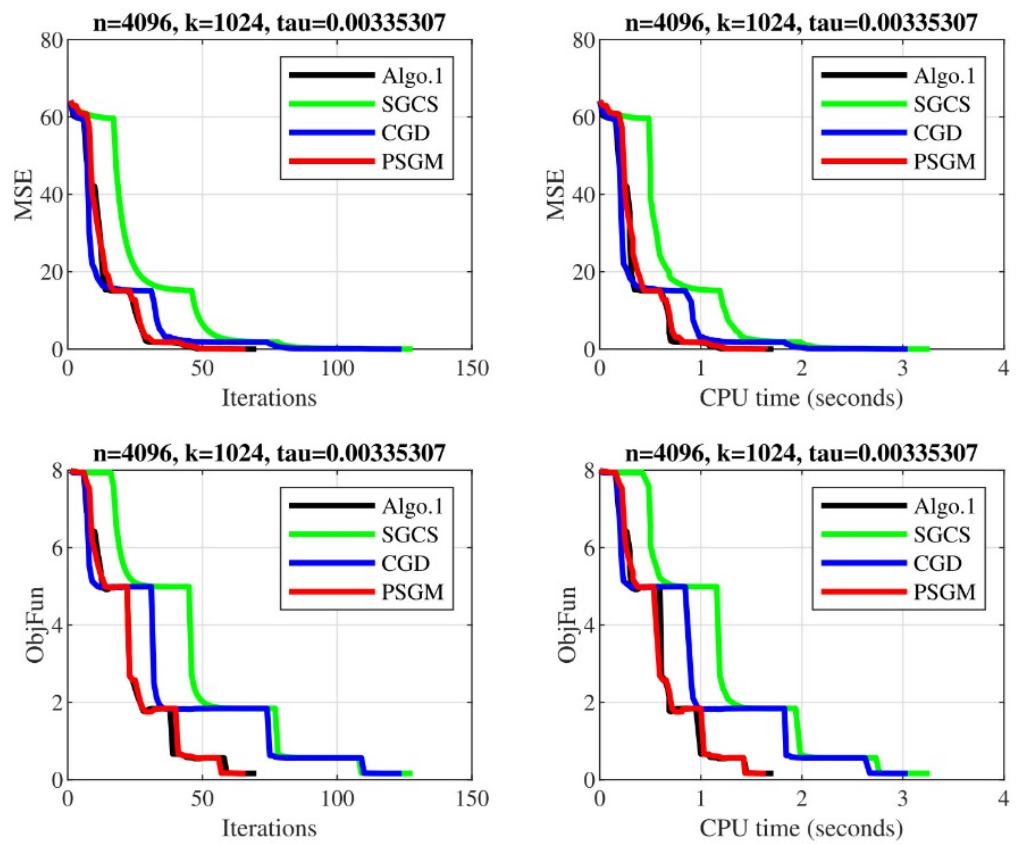

Figure 1. Comparison performance with the method Algo.1, SGCS, CGD, and PSGM. From left to right: MSE's changed trend goes hand in hand with the number of iterations or CPU time in seconds, and the changed trend in the objective function values follows the number 
Figure 1 above shows the original signal $\bar{u}$, the observed data $e$ and the signal $u^{*}$ reconstructed using the four algorithms. From figure 1 it is clear that all tested methods were able to reconstruct the signal. However, it can be observed that, Algo.1 shows to be more efficient in reconsructing the sparse signals. This is reflected by less MSE, number of iterations and CPU time. Figure 2 illustrates the change trend of MSE and objective function values in terms of number of iterations and respective CPU time. To further highlight the efficiency of the Algo.1, we repeat the experiment ten more times. Each time the experiment is repeated, Algo.1 outperforms the compared methods. From Table 1 below, we have reported the numerical result for the ten experiments. On the average, Algo.1 require 76.9 iterations to recover the sparse signal, SGCS, CGD and PSGM requires an average of 128.7, 122 and 107.9 iterations respectively. For the CPU time, Algo.1 was the fastest in reconstructing the sparse signal. Algo.1 recorded an average CPU time of 2.148 seconds while SGCS, CGD and PSGM reconstructed the sparse signal at an average of $3.639,3.505$ and 3.092 seconds respectively. Finally, with respect to the quality of recontruction, Algo.1 recorded less mean squared error compared to SGCS, CGD and PSGM.

Table 1. The experimental results of compressed sensing problem via Algo.1, SGCS, CGD and PSGM method

\begin{tabular}{ccccccccccccc}
\hline \multicolumn{4}{c}{ Algo.1 } & \multicolumn{4}{c}{ SGCS } & \multicolumn{3}{c}{$C G D$} & \multicolumn{3}{c}{$P S G M$} \\
\hline ITER & TIME & MSE & ITER & TIME & MSE & ITER & TIME & MSE & ITER & TIME & MSE \\
79 & 2.09 & $2.93 \mathrm{E}-06$ & 127 & 3.63 & $3.49 \mathrm{E}-06$ & 129 & 3.48 & $2.92 \mathrm{E}-06$ & 111 & 3.06 & $2.81 \mathrm{E}-06$ \\
74 & 2.27 & $3.15 \mathrm{E}-06$ & 131 & 4.09 & $3.63 \mathrm{E}-06$ & 117 & 3.7 & $3.03 \mathrm{E}-06$ & 103 & 4 & $1.08 \mathrm{E}-05$ \\
72 & 1.98 & $2.98 \mathrm{E}-06$ & 117 & 3.14 & $3.89 \mathrm{E}-06$ & 101 & 2.72 & $3.27 \mathrm{E}-06$ & 104 & 2.77 & $3.19 \mathrm{E}-06$ \\
75 & 1.94 & $2.86 \mathrm{E}-06$ & 132 & 3.23 & $3.33 \mathrm{E}-06$ & 104 & 2.64 & $2.45 \mathrm{E}-05$ & 114 & 2.92 & $2.64 \mathrm{E}-06$ \\
81 & 2.45 & $3.01 \mathrm{E}-06$ & 131 & 4.17 & $4.04 \mathrm{E}-06$ & 151 & 4.69 & $3.45 \mathrm{E}-06$ & 113 & 3.44 & $3.23 \mathrm{E}-06$ \\
73 & 1.88 & $2.78 \mathrm{E}-06$ & 127 & 3.27 & $3.20 \mathrm{E}-06$ & 112 & 2.94 & $3.56 \mathrm{E}-06$ & 116 & 3.05 & $2.60 \mathrm{E}-06$ \\
Average & 76.9 & 2.148 & $2.98 \mathrm{E}-06$ & 128.7 & 3.639 & $3.68 \mathrm{E}-06$ & 122 & 3.505 & $6.44 \mathrm{E}-06$ & 107.9 & 3.092 & $4.43 \mathrm{E}-06$ \\
\hline
\end{tabular}

\section{Conclusion}

This paper presents a spectral gradient projection algorithm to solve the regularized problems with the $\ell_{1}$-norm for reconstruction of sparse signal in compressive sensing. The method combines the line search method with a spectral parameter computed as a convex combination of two different spectral parameters of some conjugate gradient methods. Furthermore, we have shown that the proposed mehtod converges globally. To highlight the details of our contribution, we have presented numerical experiment in recovery sparse signal. These experiments illustrate clearly the effectiveness of our approach in reconstructing sparse signal in compressive sensing.

\section{Acknowledgments}

The second author was financially supported by Rajamangala University of Technology Phra Nakhon (RMUTP) Research Scholarship.

\section{References}

Abubakar, A. B. \& Kumam, P. (2018). An improved three-term derivative-free method for solving nonlinear equations. Computational and Applied Mathematics, 37(5), 6760-6773. https://doi.org/10.1007/s40314-018-0712-5

Abubakar, A. B. \& Kumam, P. (2019). A descent Dai-Liao conjugate gradient method for nonlinear equations. Numerical Algorithms, 81(1), 197-210. https://doi.org/10.1007/s11075-018-0541-z

Abubakar, A. B., Kumam, P. \& Mohammad, H. (2020). A note on the spectral gradient projection method for nonlinear monotone equations with applications. Computational and Applied Mathematics, 39(2), 129. https://doi.org/10.1007/s40314-020-01151-5 
Abubakar, A. B., Rilwan, J., Yimer, S. E., Ibrahim, A. H. \& Ahmed, I. (2020). Spectral three-term conjugate descent method for solving nonlinear monotone equations with convex constraints. Thai Journal of Mathematics, 18(1), 501-517.

Amini, K., Faramarzi, P. \& Pirfalah, N. (2019). A modified Hestenes-Stiefel conjugate gradient method with an optimal property. Optimization Methods and Software, 34(4), 770-782. https://doi.org/10.1080/10556788.2018.1457150

Beck, A. \& Teboulle, M. (2009). A fast iterative shrinkage-thresholding algorithm for linear inverse problems. SIAM Journal on Imaging Sciences, 2(1), 183-202. https://doi.org/10.1137/080716542

Figueiredo, M. A. T., Nowak, R. D. \& Wright, S. J. (2007). Gradient projection for sparse reconstruction: Application to compressed sensing and other inverse problems. IEEE Journal on Selected Topics in Signal Processing, 1(4), 586-597. https://doi.org/10.1109/JSTSP.2007.910281

Hale, E. T., Yin, W. \& Zhang, Y. (2007). A Fixed-Point Continuation Method for 1 -Regularized Minimization with Applications to Compressed Sensing. CAAM TR07-07, Rice University, 43, 44.

Huang, S. \& Wan, Z. (2017). A new nonmonotone spectral residual method for nonsmooth nonlinear equations. Journal of Computational and Applied Mathematics, 313, 82-101. https://doi.org/10.1016/j.cam.2016.09.014

Ibrahim, A. H., Garba, A. I., Usman, H., Abubakar, J. \& Abubakar, A. B. (2019). Derivative-free RMIL conjugate gradient algorithm for convex constrained equations. Thai Journal of Mathematics, 18(1).

Ibrahim, A. H., Kumam, P., Abubakar, A. B., Abubakar, J. \& Muhammad, A. B. (2020). Least-Square-Based Three-Term Conjugate Gradient Projection Method for \$ell_1\$-Norm Problems with Application to Compressed Sensing. Mathematics, 8(4), 602. https://doi.org/https://doi.org/10.3390/math8040602

Ibrahim, A. H., Kumam, P., Abubakar, A. B., Jirakitpuwapat, W. \& Abubakar, J. (2020). A hybrid conjugate gradient algorithm for constrained monotone equations with application in compressive sensing. Heliyon, 6(3), e03466. https://doi.org/10.1016/j.heliyon.2020.e03466

Khoramian, S. (2012). An iterative thresholding algorithm for linear inverse problems with multi-constraints and its applications. Applied and Computational Harmonic Analysis, 32(1), 109-130. https://doi.org/10.1016/j.acha.2011.03.004

Xiao, Y., Wang, Q. \& Hu, Q. (2011). Non-smooth equations based method for $\ell 1$-norm problems with applications to compressed sensing. Nonlinear Analysis, Theory, Methods and Applications, 74(11), 3570 3577. https://doi.org/10.1016/j.na.2011.02.040

Xiao, Y. \& Zhu, H. (2013). A conjugate gradient method to solve convex constrained monotone equations with applications in compressive sensing. Journal of Mathematical Analysis and Applications, 405(1), 310-319. https://doi.org/10.1016/j.jmaa.2013.04.017

Yuan, G., Li, T. \& Hu, W. (2020). A conjugate gradient algorithm for large-scale nonlinear equations and image restoration problems. Applied Numerical Mathematics, 147, 129-141. https://doi.org/10.1016/j.apnum.2019.08.022

\section{Copyrights}

Copyright for this article is retained by the author(s), with first publication rights granted to the journal.

This is an open-access article distributed under the terms and conditions of the Creative Commons Attribution license (http://creativecommons.org/licenses/by/3.0/). 